\title{
MS-ACCESS COMO FERRAMENTA DE AUXÍLIO NA ESTIMATIVA DE CUSTOS DE PROJETOS ELABORADOS NO SOFTWARE REVIT®
}

\author{
Akiro Meneses Chikushi-akiro.chikushi@fapce.edu.br \\ Engenheiro Civil formado pela UFPE e especialista em Gerenciamento de Obras pela FJN \\ Juazeiro do Norte - Ceará
}

Cícero Emericiano da Silva Junior - cicero-emericiano@hotmail.com

Engenheiro Civil pela Faculdade Paraíso do Ceará, pós-graduando em Gerenciamento da Construção Civil pela Universidade Regional do Cariri

Juazeiro do Norte - Ceará

Tiago Silva Moreira - tiagomoreiraengenharia@gmail.com

Centro Universitário Paraíso

Juazeiro do Norte - Ceará

\section{Resumo}

A elaboração de um orçamento de referência trata-se de uma estimativa dos custos de mão de obra, materiais e equipamentos que serão utilizados na construção de um empreendimento. Dessa forma, pode estar sujeito a imprecisões e assim com a possibilidade de obter superfaturamento ou até mesmo um subfaturamento. Com a utilização da tecnologia BIM, consegue-se otimizar, automatizar e garantir um produto com mais qualidade. Esse artigo tem como objetivo aplicar a tecnologia BIM 5D a um modelo de residência popular térrea para estimativa do custo de construção, através do software Autodesk Revit@ correlacionado ao MS-Access. A modelagem 3D foi executada pelo método de paredes cebolas(com elementos independentes) para que os quantitativos obtivessem um resultado preciso. $\mathrm{O}$ processo conseguiu implantar de forma rápida e confiável todos os custos dos elementos modelados no software Revit ${ }^{\circledR}$ e assim realizou-se a criação de tabelas orçamentarias. Diante dos resultados do estudo de caso realizado foi possível identificar vantagens, como resultados quantitativos precisos, uma tabela de custo mais assertiva e, como desvantagens, tempo prolongado de modelagem e incapacidade de gerar um orçamento em uma única tabele e com os custos indiretos.

Palavras-chave: Autodesk Revit $\AA^{\circ}$, BIM 5D , MS-Access.

\section{$M S$-ACCESS AS A TOOL TO ASSIST THE COST ESTIMATION OF PROJECTS MODELED IN REVIT® SOFTWARE}

\section{Abstract:}

A reference budget is an cost estimation of labor, materials and equipment that will be used in the construction of an building. Thus, it may be subject to inaccuracies and thus with the 
possibility of overpricing or even under-invoicing. With the use of BIM technology, it is possible to optimize, automate and guarantee a product with more quality. This article aims to apply BIM 5D technology to a popular single-storey residence model to estimate construction costs, using Autodesk Revit ${ }^{\circledR}$ software correlated to MS-Access. The 3D modeling was performed by the onions wall method (with independent elements) so that the quantitative ones obtained an accurate result. The process was able to quickly and reliably implement all the costs of the elements modeled in the Revit ${ }^{\circledR}$ software, thus creating budget tables. In view of the results of the case study carried out, it was possible to identify advantages, such as precise quantitative results, a more assertive cost table and, as disadvantages, prolonged modeling time and inability to generate a single table with indirect and direct costs.

Keywords: Autodesk Revit®, BIM 5D, MS-Access.

\section{INTRODUÇÃO}

As exigências por projetos cada vez mais complexos por parte dos consumidores faz a construção civil passar por um período de revolução tecnológica, visando a redução de desperdícios, melhoria na qualidade final de produtos e empreendimentos mais eficientes energeticamente Os autores Cândido e Abreu (2002, p. 3) são bem explícitos quando enfatizam que, "as questões tecnológicas surgem em função de um determinado problema a ser resolvido e/ou uma determinada necessidade a ser superada, envolvendo tanto conhecimento básico, quanto vivências práticas."

Uma das tecnologias que vem ganhando força no mercado da construção civil é o BIM (do inglês, Building Information Modelling). Segundo os autores Isikdag e Underwood (2010), BIM é a construção de modelos digitais com informações que podem ser compartilhadas com interoperabilidades, possibilitando o gerenciamento do ciclo de vida (da concepção à demolição) do empreendimento.

Um dos parâmetros extremamente relevante contido dentro desse ciclo citado e que se posiciona como antecedente ao início de qualquer construção é o estudo de viabilidade econômica, alcançado através de um orçamento. De acordo com Coelho (2001), um orçamento deve ser apresentado em uma planilha compondo seu código, descrição, unidade, quantidade, custo unitário e valor total, bem como apresentando toda sua composição de custos unitários.

Prosseguindo a observação dos comentos citados pelos autores anteriormente, percebese, significativamente, a necessidade de uma otimização nos padrões antigos de mensuração das quantidades dos insumos de um projeto, assim como, de processos mais precisos, evitando, dessa forma, estimativas errôneas de custos ocasionadas por falhas em levantamentos de quantitativos. Isto posto, considerando que o Autodesk Revit ${ }^{\circledR}$ é um software BIM, no qual carrega informações no seu modelo 3D e, sendo o MS-Access ${ }^{\circledR}$ um gerenciador de Banco de Dados, surgiu-se a motivação do presente trabalho na busca da concepção de procedimentos capazes de otimizar o gerenciamento das informações contidas na modelagem, especificamente para a área da orçamentação, no propósito de alcançar maior confiabilidade e qualidade dos resultados, reduzindo riscos e tempo na estimativas de custos dos projetos.

Devido a essa importância, o trabalho tem como objetivo geral, estudar a viabilidade técnica da inter-relação entre o MS-Access ${ }^{\circledR}$ e o software Revit ${ }^{\circledR}$ como ferramenta auxiliar no desenvolvimento de orçamentos de obras e, como objetivos específicos, realizar a quantificação de materiais do Modelo 3D elaborado no software Revit ${ }^{\circledR}$; estudar a forma de inserção de custos diretamente no Revit ${ }^{\circledR}$ por meio do MS-Access ${ }^{\circledR}$; produzir rotina otimizada de filtragem e manuseio de dados vinculados entre MS-Access ${ }^{\circledR}$ e Revit ${ }^{\circledR}$. 


\section{FUNDAMENTAÇÃO TEÓRICA}

\subsection{BUILDING INFORMATION MODELING}

A fundamentação principal do BIM é a produção de um modelo tridimensional capaz de gerenciar informações pertinentes a todo o ciclo de vida de determinado empreendimento. $\mathrm{Ou}$ seja, os elementos modelados não configuram apenas desenhos e formas geométricas, mas também, um banco de dados diversificado. Dessa forma, na metodologia BIM, criar formas gráficas que representem o projeto corretamente deixa de ser o foco principal, uma vez que depois do processo de modelagem o empreendimento já está "construído" virtualmente e todos os elementos ou representações do projeto são modificados automaticamente através da alteração de seus parâmetros. (EASTMAN et al, 2014)

Felizmente esse cenário está se revertendo através de estratégias de disseminação do BIM no Brasil e, recentemente, a assinatura do decreto $\mathrm{n}^{\circ} 10.306$ em 2 de abril de 2020, assim, estabelece que as fases de implantação se darão em intervalos de 4 anos na execução de serviços de engenharia e de obras contratadas por entidades da administração pública federal, de forma direta ou indireta. (BRASIL, 2020)

\subsection{SOFTWARE AUTODESK REVIT®}

Podemos afirmar que o Autodesk Revit ${ }^{\circledR}$ é uma das ferramentas mais conhecidas atualmente que utilizam o conceito BIM, nele é possível criar virtualmente de forma multidisciplinar e colaborativa diversos tipos de projeto de um empreendimento. Dessa maneira, com a parametrização dos elementos no projeto é possível antever interferências, levantar quantitativos, analisar custos e diversas verificações personalizadas, tudo de forma visual e interativa, criando documentos vinculados ao modelo. (NETTO, 2017).

Os componentes no Revit@ são divididos em famílias e categorias, os quais podem ser controlados por meio da inserção ou modificação de seus parâmetros sejam eles de tipo ou instância. Para uma organização e manipulação funcional, todas essas informações ficam arquivadas nos próprios componentes, estando assim disponíveis para uma posterior filtragem, extração e visualização em tabelas.

Através da criação de tabelas no Revit® é possível levantar quantitativos e informações relevantes do projeto e também realizar análises importantes do modelo criado. Fica claro que o gerenciamento de informações em tabelas no Revitß só é possível devido a parametrização dos elementos, então quanto mais parâmetros forem adicionados, mais possibilidades de análises são disponibilizadas. (WHITBREAD, 2016)

\subsection{NÍVEL DE DETALHAMENTOS DE PROJETOS EM BIM}

Segundo a definição do manual LOD Specification, disponibilizado pelo BIMFORUM (2018), no qual submete-se a padrões do protocolo do AIA(Instituto Americano de Arquitetura), o LOD (Level of Development), traduzido como Nível de desenvolvimento, é uma classificação gerada com a intenção de padronizar, de forma organizada, as etapas do desenvolvimento de um determinado projeto em BIM, onde cada uma delas carregam níveis de informações e confiabilidade desses dados. Compondo o nível de desenvolvimento, estão inclusos o nível de detalhamento(Lod - acrônimo de Level of Detail) e o nível de informação(LOI - acrônimo de Level of Information). De uma forma geral, a modelagem apresenta tanto sua representação gráfica quanto informações das características do objeto, 
enumeradas, sequencialmente, onde valores maiores caracterizam maiores graus de precisão e confiabilidade do modelo, conforme demonstrado na figura 01 e descrições abaixo.

Figura 01 - Exemplos gráficos de LOD

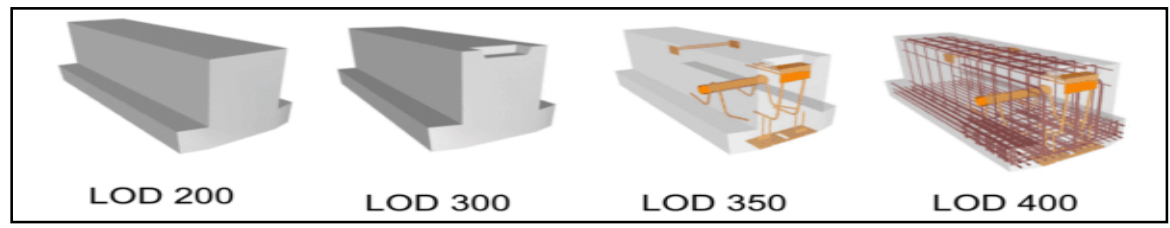

Fonte - Adaptado de BIMFORUM(2018)

O supracitado material denota cada nível de LOD referenciando-os às suas fases de projetos/execução, como o apresentado a seguir: LOD 100 (conceito), LOD 200 (desenvolvimento do design), LOD 300 (documentação), LOD 350 (coordenação), LOD 400 (construção) e LOD 500 (Gerenciamento e manutenções). Reforçando e demonstrando, pelos seus modelos, a importância da padronização de determinados processos de modelagem.

\section{METODOLOGIA}

A elaboração da metodologia do presente artigo partiu da problemática: "Seria possível a utilização do software MS-Access ${ }^{\circledR}$ para manipulação do Banco de Dados existente no software Autodesk Revit ${ }^{\circledR}$ facilitando, assim, a elaboração de Orçamentos de obras?" Dentro de tal proposta, a pesquisa adotou, conforme os seus objetivos, a exploratória e de laboratório, envolvendo desde levantamentos bibliográficos, onde utilizou-se de fontes de informações tais como: arquivos de plantas, normas, conteúdo da internet, e uma seleção documental, sobre o tema analisado, até o procedimento de estudo de caso e simulações experimentais necessários para se chegar às conclusões. Através dos procedimentos, se buscou analisar a implantação do MS-ACCESS em um projeto de uma residência unifamiliar real, onde foram inseridas informações externas de custos, como forma de testes e, ademais ao exposto, a natureza da pesquisa abrangeu uma avaliação qualitativa, de modo a comprovar a viabilidade técnica do estudo.

\subsection{CARACTERIZAÇÃO DO OBJETO DE ESTUDO}

O imóvel analisado no estudo refere-se a uma residência unifamiliar, padrão normal, modelada no software Revit ${ }^{\circledR}$, cuja área construída equivale à $60,99 \mathrm{~m}^{2}$. As análises e resultados considerados abrangeram apenas a disciplina de arquitetura, com modelagem classificada em nível de desenvolvimento e detalhamento equivalente ao LOD 350, conforme pode ser visto nas figuras 02 e 03 .

Figura 02 - Planta Baixa

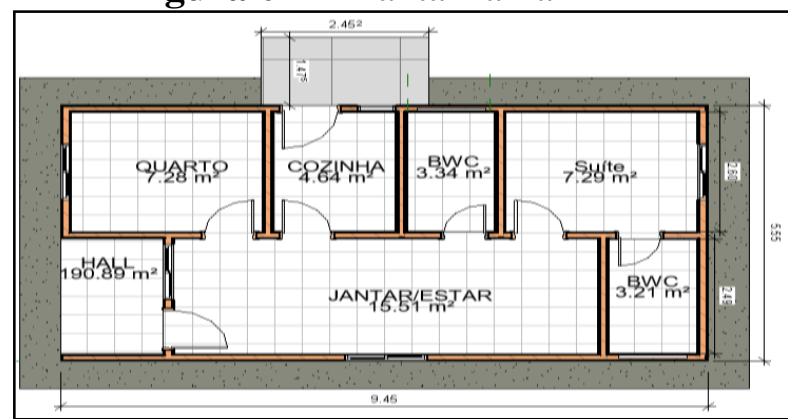

Fonte - Acervo dos Autores(2020)
Figura 03 - Modelagem em LOD 350

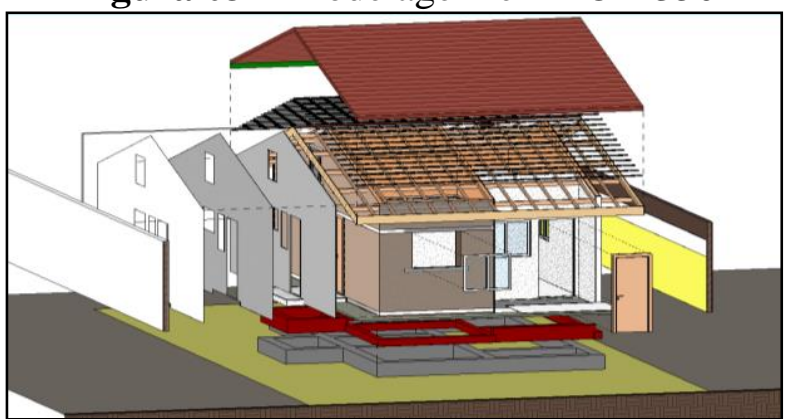

Fonte - Acervo dos Autores(2020) 


\subsection{INSERÇÃO DE CÓDIGOS DE CUSTOS NO SOFTWARE REVIT®}

Para tornar possível o estudo de viabilidade de manipulação do banco de dados do Revit ${ }^{\circledR}$ através do MS-Access, houve a inclusão dos códigos da tabela de referências de custos unitários SEINFRA-CE, versão 026.1, nos elementos do projeto modelo para, dessa forma, possibilitar posteriormente a assimilação com seus respectivos custos, iniciando, assim, as primeiras análises. A forma encontrada para se obter êxito no carregamento dessas informações externas para dentro do software, foi a utilização da extensão .txt e geração de lista formatada seguindo padrões sequenciais do comando 'nota-chave'. A figura 04 mostra, ao lado esquerdo, toda a codificação da tabela SEINFRA-CE inserida e, ao lado direito, itens da modelagem associados aos seus códigos indicativos.

Figura 04 - Códigos inseridos no Revit ${ }^{\circledR}$

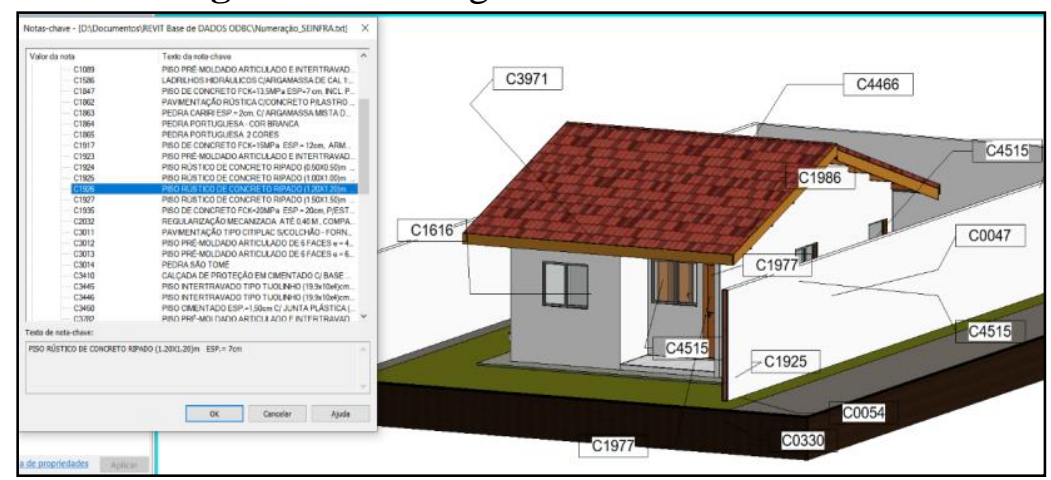

Fonte - Acervo dos Autores(2020)

O procedimento adotado nesse processo foi correlacionar os códigos com os materiais, através do campo de 'nota-chave', existente dentro da janela de 'materiais' do software Revit ou pelo comando 'nota-chave', localizado dentro do menu 'Anotar', como exibido na figura 05

Figura 05 - Janela 'Materiais'(lado esquerdo) e Comando 'Anotar'(lado direito)

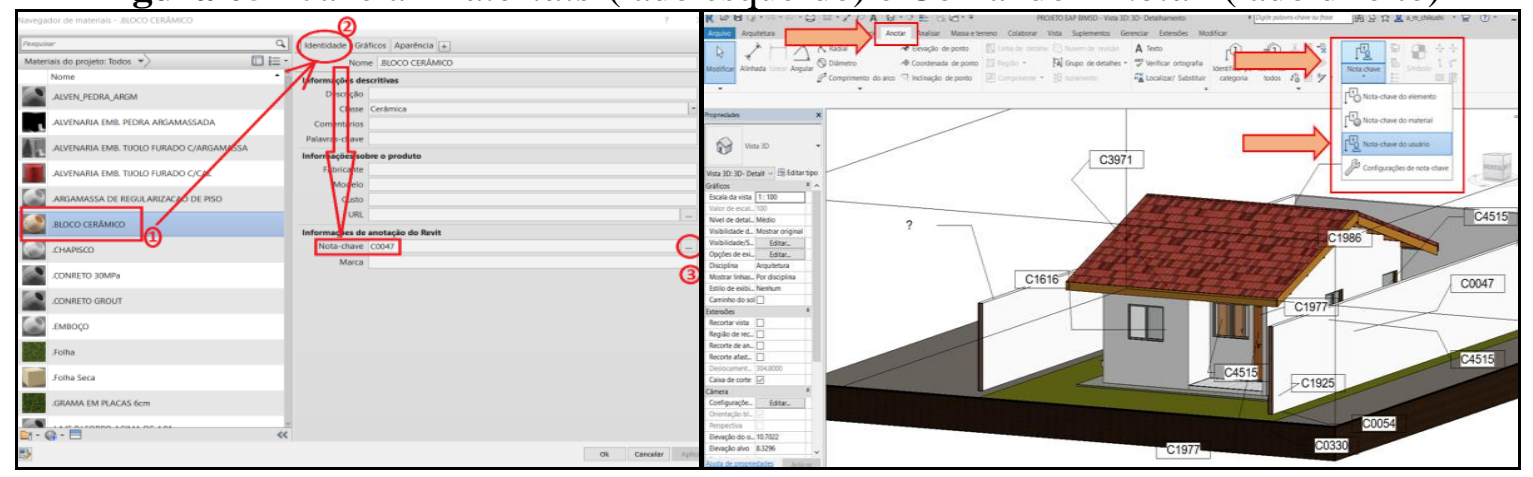

Fonte - Acervo dos Autores(2020)

\subsection{CONEXÃO REVIT®/MS-ACCESS E MANUSEIO DE INFORMAÇÕES}

Para interligação entre os dois softwares, optou-se pela utilização do add-on 'DB Link' cujo desígnio da ferramenta é a importação/exportação do banco de dados do projeto com o software Access ${ }^{\circledR}$. A figura 06 expõe o comando supracitado. 
Figura 06 - Vinculação com o MS-ACCESS pelo Add-On DBLINK

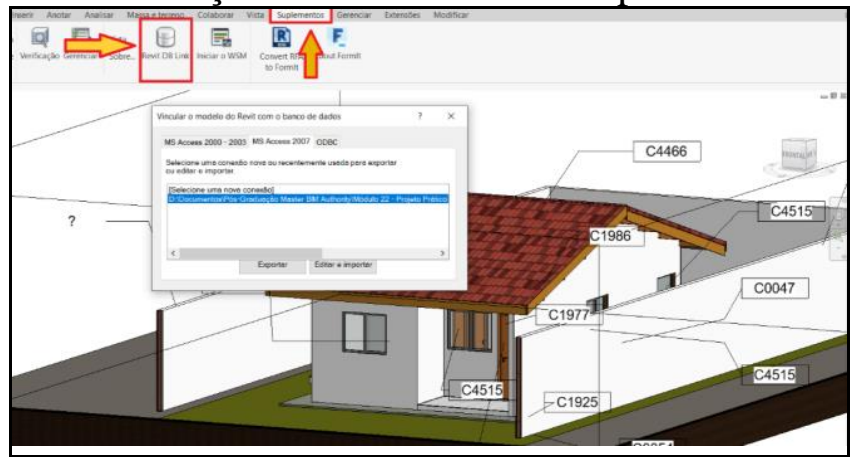

Fonte - Acervo dos Autores(2020)

\section{ANÁLISE DE DADOS E RESULTADOS}

Ao recolher o banco de dados advindo do projeto, o Ms-Access separa por tabela de classes todos os elementos contidos no arquivo do Revit $^{\circledR}$, independentemente de terem sido utilizados na modelagem 3D. Por exemplo: uma tabela específica para colunas estruturais é gerada, mesmo que não tenha sido inserido nenhum pilar no projeto, assim como também, tabela de dutos de ar-condicionado, luminárias, esquadrias etc. Em suma, o procedimento de importação/exportação engloba uma extensa e complexa gama de subsídios, demonstrando ser uma potente ferramenta para sincronização entre os dois programas.

A partir disso, consultas são prontamente elaboradas entre as tabelas levantadas, possibilitando uma busca delineada pelos códigos inseridos no projeto e correlacionando-os com itens da tabela SEINFRA-CE previamente cadastrada no MS-Access. A figura 07 menciona, na coluna $\mathrm{A}$, as diversas tabelas de classes compartilhadas na rotina; nas colunas B1 e B2, os parâmetros relacionados à descrição, respectivamente, dos itens da tabela SEINFRA-CE e do projeto; e nas colunas $\mathrm{C} 1$ e $\mathrm{C} 2$, variáveis referentes aos custos, respectivamente, dos itens da tabela SEINFRA-CE e do projeto, sendo perceptível estarem vazios os campos de B2 e C2.

Figura 07 - Filtragem e analogia de dados importados

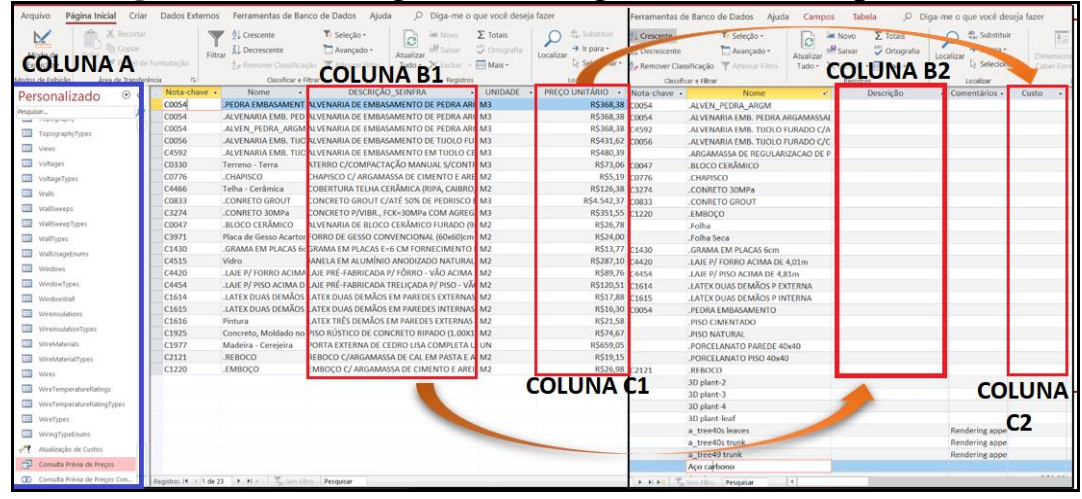

Fonte - Acervo dos Autores(2020)

Como é possível observar, a associação adotada integra, respectivamente, campos dos materiais do software Revit $^{\circledR}$ com itens da tabela SEINFRA-CE, atrelando os componentes Nota-Chave com o Código, Comentários com Unidade, Descrição como Descrição e Custo com Preço Unitário, logrando, de forma automática e segura, a atualização aspirada.

Concluída essa primeira etapa, realiza-se a transmissão das novas informações atualizadas do Access para o software Revit ${ }^{\circledR}$ pelo mesmo aplicativo, no caso, o DB Link, ao 
qual preencherá com esses valores os indicadores contidos na aba de identidade dos materiais, consoante ao explanado na figura 08 .

Figura 08 - Dados atualizados de identidade dos materiais

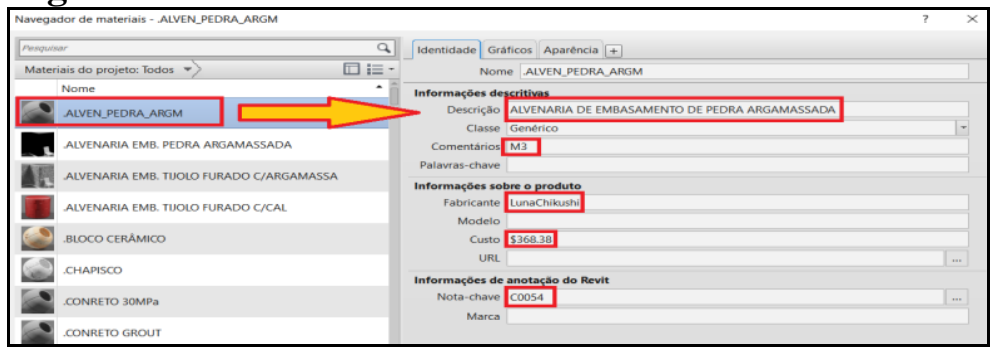

Fonte - Acervo dos Autores(2020)

A comprovação de que todos as informações pleiteadas têm sido atribuídas dentro do sistema do Revit ${ }^{\circledR}$ é demonstra pelo levantamento de materiais e custos contidas na Figura 09.

Figura 09 - Comparação antes/depois da Tabela de Estimativa de custos

\begin{tabular}{|c|c|c|c|c|c|c|c|c|c|}
\hline NOTACHN & DESCRRÇCंO & UNDODEE & Custo & Moterial Nome & NOTACHAV & DESCRIC,AO & UNIDADE & CUSTo & Material Nome \\
\hline & & & 50.00 & & & & & 80.00 & \\
\hline $\cos 21$ & & & 80.00 & BLOCO CERAMICO & coovi & ALENARIII DE BLOCO CERMAMICO FURADO I & & 526.70 & BLOCO CERMMICO \\
\hline $\cos 44$ & & & 50.00 & PEDRA EMBA SAMENTO & $\cos 4$ & AIVENARIA DE EMBASSAMENTO DE PEDRA : & & $\$ 388.38$ & PEDRA EMBA SAMENTIO \\
\hline $\cos 56$ & & & 80.00 & ALVENARIA EMB. TIJOLO FURADO CICAL & $\cos 6$ & ALVENARIA DE EMBASSMENTTO DE TUOLO & & $\$ 311.62$ & AIVENARIA EMB. TJUOLO FUARDO CICAL \\
\hline conte & & & $\$ 0.00$ & CHAPISCO & 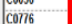 & CHAPISCO C I ARGGAMASA DE C CIMENTOE & & 55.19 & CHAPISCO \\
\hline C1220 & & & 80.00 & EMBOC, & $\frac{10120}{c 1220}$ & EMBOCOC CI ARGAMASSA DE CIMENTOE ARR & & 826.90 & 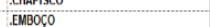 \\
\hline C1616 & & & 50.00 & Pintura & (172) & 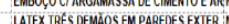 & & 86.50 & Pinous \\
\hline C1925 & & & 80.00 & Concerte, Moldado no local - Cimza & $\frac{161616}{c 1925}$ & 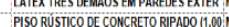 & & $\begin{array}{l}51,58 \\
514.67\end{array}$ & \\
\hline c1977 & & & 50.00 & Madeira-Cerejeira & (1925 & 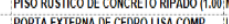 & & 514.67 & Concerto, Moddado nol local Cinza \\
\hline C22121 & & & 80.00 & REEOCOO & (1991) & PORRA EXEERRA DECEERO LSA COMP & & 5699.15 & Madeira-Cerejeira \\
\hline C2181 & & & $\$ 0.00$ & REGUUARIZACAO DE PISO & (212121 & REEOCOCAARGAMASSA DE CAL EM PASTA! & & $\$ 19.15$ & REDOCO \\
\hline c39i1 & & & 80.00 & Place de Gesso Acartonado & (3991 & 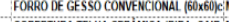 & & 524.00 & Placa de Gesso Acartonado \\
\hline C4466 & & & $\$ 0.00$ & Tella - Cerâmica & CMG6 & COBERTURA TELHA CERQMMICA (RRIPA, CABB I) & & $\$ 126.38$ & Tellha - Cerámica \\
\hline casis & & & $\$ 80.00$ & Vidro & Casis & JANELA EM aLUUMINO ANOOZZZDO NATURA: & & $\$ 887.10$ & \\
\hline
\end{tabular}

Fonte - Acervo dos Autores(2020)

Como percebido, os campos vazios DESCRIÇÃO e UNIDADES contidos dentro do contorno vermelho foram preenchidos, assim como os CUSTOS que inicialmente estavam com valor ZERO dentro das células do contorno azul, foram atualizados.

\section{CONSIDERAÇÕES FINAIS}

Perante o estudo realizado é possível concluir que o processo correlacional entre os dois softwares apresenta viabilidade técnica para inserção de dados em projetos, auxiliando, portanto, na análise de estimativa de custos. Percebe-se, também, a capacidade do processo ser meritório no gerenciamento de outros coeficientes relevantes para otimização de determinadas atividades, sejam para análise de resistência mecânica, sejam para estudos de confortos térmicos etc. Ademais, observaram-se as descritas vantagens: quantificação de materiais de forma precisa e confiável; possibilidade de alteração e manipulação de diferentes referências de preços e estimativa de custos ao passo em que o projeto é desenvolvido. Entretanto, existem algumas ponderações pertinentes a serem enfatizadas tais como: a necessidade da modelagem ser em LOD acima de 300 para se ter uma caracterização melhor dos resultados; a incapacidade de geração de um orçamento completo no software Revit ${ }^{\circledR}$ por este não conseguir gerar uma tabela única contemplando todos os itens; processo não inteiramente automático por necessitar de procedimentos manuais a cada alteração nas tabelas de preços em decorrência de cada uma possuir diferenças nos seus códigos.

Uma apuração igualmente constatada, enquanto o desenvolvimento do trabalho, concerne ao fato do Revit® não poder ser qualificado como um software de orçamentação, pela sua incapacidade de inserção de custos indiretos de obras Isso decorre porque somente objetos modelados são suscetíveis de receber as informações e, devida a essa inabilidade e limitação, 
há ainda a necessidade de envolvimento de outros programas para a finalização e detalhamento de uma proposta orçamentária completa, o que não implica descarte da proposta apresentada, por esta conseguir perfeitamente oferecer uma noção do valor construtivo do empreendimento de forma estimativa.

Dessarte, partindo dos observáveis do trabalho ora exposto, consegue-se perceber a potencialidade da conexão Revit ${ }^{\circledR} / M S$-Access ${ }^{\circledR}$ como alternativa para análises de viabilidade da construção, devida à capacidade de manipulação de tipos diversos de informações, indo exatamente de encontro ao objetivos primordiais da tecnologia BIM, que é a comunicação entre softwares. Por fim, as demonstrações corroboram o alcance dos objetivos sugeridos, demonstrando uma admirável solução alternativa para implantação de tabelas orçamentárias dentro de um modelo Revit.

\section{REFERENCIAS}

BIM FORUM. Level Of Development Specification Guide: For Building Information Models. [S. l.], 2018.

BRASIL. Decreto $\mathbf{n}^{\mathbf{0}} \mathbf{1 0 . 3 0 6}$, de 2 de abril de 2020. Estabelece a utilização do Building information Modeling na execução direta ou indireta de obras e serviços de engenharia realizada pelos órgãos e pelas entidades da administração pública federal, no âmbito da Estratégia Nacional de Disseminação do Building Information Modeling, DF,Disponível em: http://www.planalto.gov.br/ccivil_03/_ato2019-2022/2020/Decreto/D10306.htm Acesso em: 11 ago. 2020, 11:32

CÂNDIDO, G. A.; ABREU, A. F. O processo de implantação de novas tecnologias e a busca da sinergia entre indivíduo e organização. Revista de Ciências da Administração, Santa Catarina, v. 4, n. 8, p. 1-13, jul./dez. 1993.

COELHO, S. S.; NOVAES, C. C. Modelagem de Informações para Construção (BIM) e ambientes colaborativos para gestão de projetos na construção civil. [S. l.], 2008.

Disponível em:

<http://www2.pelotas.ifsul.edu.br/gpacc/BIM/referencias/COELHO_2008.pdf>. acesso em: 10/06/2019.

EASTMAN, Chuck; TEICHOLZ, Paul; SACKS, Rafael; LISTON, Kathleen. Manual de BIM: um guia de modelagem da informação da construção para arquitetos, engenheiros, gerentes, construtores e incorporadores. Porto Alegre: Bookman, 2014.

ISIKDAG, U.; UNDERWOOD, J. A Synopsis of the Handbook of Research on Building. [S. l.], p. 84-96, 2010. Disponivel em:

<https://www.researchgate.net/profile/Umit_Isikdag/publication/235759628_A_Synopsis_of_ the_Handbook_of_Research_on_Building_Information_Modelling/links/09e4151338477b9fd 2000000/A-Synopsis-of-the-Handbook-of-Research-on-Building-Information-

modelling.pdf $>$. Acesso em: 8 Maio 2019.

NETTO, Claudia. Autodesk Revit® Architecture 2016: conceitos e aplicações. 1. ed. São Paulo: Saraiva, 2017.

WHITBREAD, Simon. Mastering Autodesk ${ }^{\circledR}$ Revit ${ }^{\circledR}$ MEP 2016. 1. ed. Canadá: Sybex, 2015. 